\title{
CIRUGÍA ENDOSCÓPICA UROLÓGICA ASISTIDA POR ROBOT
}

\author{
Octavio Castillo',2, Carlos Javier Ahualli', Rafael Sánchez - Salas', Ivar Vidal', Rodrigo \\ Campos', Miguel Feria - Flores' y Francisco Sepúlveda'.
}

'Departamento de Urología. Clínica INDISA. Santiago. Chile.

${ }^{2}$ Departamento de Urología. Facultad de Medicina de la Universidad de Chile. Santiago. Chile.

\begin{abstract}
Resumen.- OBJETIVO: La evaluación comprensiva de los procedimientos quirúrgicos sigue siendo la piedra angular del control de calidad. La Cirugía Robótica se ha convertido en una popular opción quirúrgica en urología. Realizamos una revisión de la literatura médica en Inglés de Cirugía urológica endoscópica robóticamente asistida (CUERA) con el fin de evaluar las tendencias de las publicaciones médicas en relación con este tema.

MÉTODOS: La información para este estudio se obtuvo mediante búsquedas en PubMed desde el 2003 al 2008. Las palabras claves utilizadas en la búsqueda incluyeron: "cirugía urológica robótica", "cirugía endoscópica asistida por robot". Sólo artículos publicados en inglés fueron incluidos en esta revisión.
\end{abstract}

RESULTADOS: Se identificaron 383 estudios. De estos, 366 correspondían a series de artículos clínicos de diferentes temas relacionados con la robótica en urología; 13 artículos presentados realizaron comparaciones de cirugía ya sea robótica, laparoscópica o abierta en procedimientos como la cistectomía radical (CR) (1), prostatectomía radical (PR) (10) y pieloplastia (P) (2). Cuatro estudios correspondieron a pruebas de control randomizadas y abordaron telerounding (T) (2), acceso renal percutáneo (ARP) (1) y el sostén robótico de la cámara en cirugía endoscópica (SRC) (1).

CONCLUSIONES: CUERA es una nueva opción quirúrgica urológica para cirugías como la prostatectomía radical, pieloplastia y cistectomía radical. Hay información con bajo nivel de evidencia que apoye la idea de la cirugía robótica como el próximo estándar de oro quirúrgico en urología. Existe una evolución quirúrgica en favor de los posibles beneficios de la tecnología robótica, pero hay necesidad de realizar estudios prospectivos controlados y randomizados en cirugía robótica urológica con el fin de consolidar la aplicación de esta novedosa tecnología.

Palabras clave: Cirugía urológica robótica. Cirugía urológica asistida por robot.

Summary.- OBJECTIVES: Comprehensive evaluation of surgical procedures remains the cornerstone of quality control. Robotic surgery has become a popular surgical option in urology. We review the English medical li-terature on urological robot-assisted endoscopic surgery (URAES) in order to assess the trends of medical publications regarding this subject. 
METHODS: All Studies addressing URAES were included. Information for this review was obtained by Pub Med searches from 2003 to 2008 . Keywords used in our search included: "robotic urologic surgery", "robotic assisted urological surgery". Only articles published in English were included in this review.

RESULTS: We identified 383 studies. 366 articles corresponded to clinical series of different to-pics regarding robotics in urology; 13 articles presen-ted comparisons of robotic surgery versus either pure laparoscopic or open surgery in urological procedures such as radical cistectomy (1), radical prostatectomy (10) and pyeloplasty (2). Four studies corresponded to randomized control trials, addressing robotic teleroun-ding (2), kidney percutaneous access (1) and camera holding devices for endoscopic surgery (1).

CONCLUSIONS: URAES is an emerging surgical option for urological operations like radical prostatectomy, pyeloplasty and radical cystectomy. There is lack of highlevel evidence information to support the idea of robotic surgery as the upcoming surgical gold standard in urology. Surgical evolution will favour the potential benefits of robotic technology, but there is need for design and performance of prospective randomized trials in robotic urologic surgery in order to consolidate the application of this novel technology.

Keywords: Robotic urologic surgery. Robotic assisted urological surgery.

\section{INTRODUCCIÓN}

En 1988 el robot inglés "World First" fué el primero en el mundo en extraer fragmentos de tejido humano en un quirófano, concretamente en una prostatectomía, por lo que corresponde a la urología este hito de la historia de la robótica (1). Y Wang funda en 1989 la empresa Computer Motion Inc. (Goleta, CA, USA) especializada en la construcción de robots quirúrgicos, que irá desarrollando los proyectos AESOP, Hermes y Zeus (2). Hacia 1993 Computer Motion Inc, comenzó a trabajar en el robot cirujano Zeus, cuyo primer prototipo estuvo disponible en 1995. El proyecto AESOP (Automated Endoscopic System for Optical Positioning) se remonta a 1994, con el modelo 1000, que fue el primer robot del mundo aprobado por la FDA. En 1996 la empresa Computer Motion Inc. siguió con las mejoras hasta llegar al AESOP 4000, disponible hoy día (3).

En 1995 se constituye la empresa Intuitive Surgical Inc (Sunnyvale, CA, USA) con el objeto de desarrollar un proyecto completo de robótica quirúrgica (4). En 1999 surge el robot quirúrgico más avan- zado conocido hasta la actualidad, el da Vinci® Surgical System, que en el 2000 obtiene la validación de la FDA para realizar procedimientos de cirugía abdominal vía laparoscópica. La cirugía robótica de avanzada fue introducida en este año (5). Por su parte el robot PROBOT, del Imperial College of London, fue usado en el bienio 1997-98 para realizar operaciones endoscópicas de próstata en Inglaterra (6), estableciendo un hito en la robótica quirúrgica ya que se trata de un robot autónomo que trabaja después de la preprogramación sobre el caso clínico, una vez obtenidas coordenadas ecográficas.

En 1998 fué realizado el primer acceso renal percutáneo robotizado por telecirugía con PAKYRCM, entre el Johns Hopkins Hospital, de Baltimore, MA, USA, y el Hospital Tor Vergara de la Universidad de Roma, Italia (3). En septiembre de 2001 se realizó la primera colecistectomía transoceánica con el robot ZEUS, estableciendo un hito en telecirugía. El robot operó a una mujer de 68 años en Estrasburgo (Hospital Civil del Este de Francia), mientras que el equipo de cirujanos controlaba los mandos de la consola desde New York (Mount Sinai Medical Center) a $7.000 \mathrm{Km}$ de distancia (7). Hacia el 2001 la unidad de robótica del Johns Hopkins Medical Institution (Baltimore) presentó los resultados de telecirugía robótica con el robot AESOP 3000 (Brazo asistente) y PAKI-RCM (puncionador renal estereotáxico), en la reunión anual de la $A \cup A$, que preludiaban la gran afinidad entre urología, dispositivos robotizados y telecirugía. Las últimas novedades en robótica quirúrgica vienen de la mano del da Vinci Surgical System. En junio del 2000, Intuitive Surgical salió a bolsa y en 2003 adquirió a su principal competidor, Computer Motion, absorbiendo los proyectos Zeus (similar a da Vinci), AESOP y Hermes, convirtiéndose en el líder mundial de fabricantes de robots quirúrgicos. Las intervenciones con da Vinci han experimentado un aumento constante en los últimos años (3).

Como se puede observar, actualmente el campo de la cirugía y en especial el de la urología se encuentra en un gran momento de desarrollo tecnológico a través de su principal protagonista, el robot. Dentro de la última década la cirugía urológica endoscópica robóticamente asistida (CUERA) ha surgido con la promesa de extender los beneficios de la cirugía mínimamente invasiva, buscando disminuir la morbilidad y lograr la mejora de los resultados postoperatorio (8). La evaluación integral de los resultados quirúrgicos continúa siendo la piedra angular del control de calidad en la cirugía. En lo referido al campo de las comunicaciones en la atención médica, se ha producido un lento pero firme progreso. Recientemente, se ha mostrado al "telerounding $(T)^{\prime \prime}$ como un complemento de las visitas del 
médico de cabecera con resultados satisfactorios en lo que respecta a la percepción del paciente y su cuidado postoperatorio (9). Vale la pena remarcar que la principal preocupación en lo que se refiere al control médico a distancia es saber si se podrán identificar en forma oportuna las complicaciones durante el período postoperatorio (10-13). En lo que se refiere a las cirugías propiamente dichas, la prostatectomía radical laparoscópica (PRL) es una efectiva opción de tratamiento para hombres con cáncer de próstata y ofrece el control del cáncer en pacientes con enfermedad localizada $(14,15)$. Más de 35.000 prostatectomías radicales asistidas por robot (PRLAR) fueron realizadas en EEUU en el año 2005 (16-19). Recientemente, la PRLAR ha sido reconocida como una opción efectiva para los pacientes con cáncer de próstata localizado (20). Aunque es bien conocida la clara efectividad del robot en la realización de la prostatectomía radical, los costos que implica la utilización de la interface robótica, constituyen un elemento limitante. Sin embargo, CUERA también ha sido empleado en otros procedimientos urológicos como lo es la cirugía laparoscópica renal, donde la nefrectomía laparoscópica asistida por robot (NLAR) tanto total (NRLAR) como parcial (NPLAR), ha sido realizada de manera efectiva y prometedora $(21,22)$. En el caso especifico del cáncer invasor de vejiga, la Cistectomía Radical abierta (CRA) continúa siendo el estándar de oro para el tratamiento quirúrgico. Sin embargo, los esfuerzos por reducir la morbilidad en la CR han fomentado el interés en los procedimientos con mínimo acceso. En este sentido la cirugía robótica ha sido igualmente empleada en cistectomías y los resultados iniciales muestran probables beneficios que deberán ser verificados a largo plazo (23-27). Entre las principales preocupaciones acerca de la CR con mínimo acceso podríamos citar el aumento de los costos y la duración de las cirugías, el potencial limitado para una linfadenectomía, y lo que es más importante, una ausencia de resultados oncológicos y funcionales a largo plazo (28).

Cabe mencionar además que la robótica permitió al cirujano poder realizar complejos procedimientos reconstructivos como lo son las pieloplastias desmembradas y no desmembradas (P) (29).

Otros procedimientos descritos en la literatura y que son objeto de nuestro análisis son los accesos renales percutáneos telerobóticos (ARR) (45).

En todos estos procedimientos quirúrgicos con mínimo acceso citados anteriormente el cirujano, idealmente, debe manipular la cámara y esto conduce muchas veces a problemas de comunicación y disturbios durante la cirugía que son causados por el movimiento. El sostén robótico de la cámara (SRC) en cirugía endoscópica permitió al cirujano estabilizar la imagen laparoscópica y así reducir al mínimo estas complicaciones (30). Actualmente, hay limitaciones en la presentación de la literatura e investigación acerca del uso de la robótica en Urología.

El presente estudio busca definir la situación actual de la calidad de la literatura concerniente a la aplicación de la cirugía robótica en el campo de la Urología. Consideramos que solo una información con elevado nivel de evidencia podría consolidar la versión robótica de las cirugías urológicas y por tanto establecerlas como un estándar de tratamiento.

\section{MATERIAL Y MÉTODOS}

La información para este estudio se obtuvo mediante búsqueda de publicaciones en Pub Med de entre 2003 al 2008. Las mismas se hicieron con restricción de idioma (solamente artículos publicados en Ingles fueron incluidos en esta revisión). Las palabras claves utilizadas incluyeron: "robotic urologic surgery", "robotic assisted urological surgery". Consideramos que la búsqueda debía ser realizada en Ingles, por ser en esta lengua que se difunde la mayor parte del conocimiento científico. Además existe un escaso número de publicaciones sobre el tema en el idioma español.

\section{RESULTADOS}

Se identificaron 383 estudios. De estos, 366 correspondían a series de artículos clínicos de diferentes temas relacionados con la robótica en urología; 13 artículos presentados realizaron comparaciones de cirugía ya sea robótica, laparoscópica o abierta en procedimientos como la cistectomía radical (1), prostatectomía radical (10) y pieloplastia (2). Cuatro estudios correspondieron a pruebas de control randomizadas y abordaron telerounding (2), acceso renal percutáneo (1) y el sostén robótico de la cámara en cirugía endoscópica (1).

\section{Telerounding}

En nuestra búsqueda encontramos 2 estudios controlados y randomizados que compararon la tradicional visita médica con el T $(31,32)$. En ambos casos se estudió el grado de respuesta al cuestionario de evaluación y satisfacción del paciente en el postoperatorio, el tiempo de estadía y la morbilidad. Los resultados demostraron un alto rango de respuesta (100\%). El rango de morbilidad fue similar en ambos estudios (13-16\%) al igual que el tiempo de estadía. Hubo un incremento en la satisfacción del paciente en el cuidado postoperatorio con T en comparación 
con la tradicional visita en lo referido al rigor del examen, calidad de información medica, coordinación de los cuidados postoperatorios y disponibilidad del médico tratante.

\section{Prostatectomía radical}

En nuestra búsqueda 10 estudios presentaron algún tipo de comparación entre cirugía robótica versus cirugía abierta o laparoscopica pura. En la mayoría de ellos el principal objetivo fue comparar la Prostatectomía radical abierta (PRA) vs PRL y PRLAR (33-39). Los resultados obtenidos mostraron que la PRL y PRLAR disminuyen la convalecencia, estadía hospitalaria, dolor postoperatorio, pérdidas sanguíneas, transfusiones y complicaciones con respecto a la PRA (38-40). Las diferencias entre las pérdidas sanguíneas y los tiempos quirúrgicos entre la PRL y la PRLAR fueron evaluadas en tres estudios $(34,39,41)$. En cuanto a la continencia y función sexual no se reportaron diferencias significativas entre la PRA y la PRL y PRLAR (33). En cuatro estudios se evaluó las diferencias existentes entre PRL y PRLAR $(34,39,41,42)$. Se demostró disminución de los márgenes positivos órganos confinados pero resultados muy semejantes en la serie total comparando PRA vs PRL y PRLAR (36). Cabe mencionar que la evaluación de los resultados oncológicos y funcionales fueron evaluados a corto plazo (35-37).

\section{Cistectomía radical}

Un estudio comparó el perioperatorio de los pacientes sometidos a una CRA y la cistectomía radical robótica (CRR) (43). Las complicaciones fueron similares en ambos grupos, con disminución de las pérdidas sanguíneas, de las transfusiones y la estadía hospitalaria en el grupo de CRR pero incrementó los tiempos quirúrgicos. Los resultados en lo referido a los márgenes positivos y el número de nódulos linfáticos resecados fué similar favoreciendo levemente al grupo de la CRA.

\section{Pieloplastia}

Dos artículos encontramos en nuestra búsqueda $(29,44)$. Ninguno de ellos presentaba criterios de control ni randomización. Los resultados observados demuestran resultados estadísticamente similares en cuanto al perioperatorio. Sin embargo los tiempos quirúrgicos si mostraban diferencias al igual que los costos. El tiempo medio y tiempo total de cirugía en el caso de la pieloplastia laparoscópica robóticamente asistida (PLRA) fué significativamente más largo que la pieloplastia laparoscópica (PL) por 19,5 y 39,0 minutos respectivamente. PLRA era mucho más costosa que el PL (2,7 veces), debido a que el largo tiempo operatorio conducía al aumento de los gastos de consumo. El análisis mostró además que el tiempo quirúrgico de la PL debería aumentar a casi 6,5 horas para que se convierta en costo equivalente a PLRA. Si bien la experiencia clínica inicial con robot es favorable (29), este estudio más reciente muestra que para el experimentado laparoscopista, la aplicación actual del robot no dá lugar a un beneficio clínico significativo con sustancial costo.

\section{Nefrolitotomía percutánea}

El estudio controlado y randomizado encontrado en nuestra serie comparó el acceso renal percutáneo durante una nefrolitotomía realizado por el cirujano y el acceso telerobótico. El mismo demostró un tiempo medio para completar la inserción de la aguja 35 segundos para el humano comparado con una media de 57 segundos para el robot. Si bien el robot fué más lento como se observó en la serie el mismo fué más efectivo, $88 \%$, comparado con 79 $\%$ de efectividad para el cirujano durante el primer intento (45).

\section{Sostén Robótico de la Cámara en cirugía en- doscópica}

Solo un estudio era controlado y randomizado (46). El mismo comparó 2 tipos de robot durante la PRL: EndoAssist y AESOP y evaluó el tiempo quirúrgico y accionar durante la prostatectomia radical laparoscopica. Mostraba que ambos sistemas presentaban una eficacia similar con respecto a la performance durante la PRL. Los tiempos de preparación favorecieron al AESOP $(2.0$ min contra 5.3 $\mathrm{min}$ ). En cuanto a los tiempos de disección de los conductos deferentes y las vesículas seminales favorecieron al EndoAssist (23 min contra $33 \mathrm{~min}$ ). Sin embargo no se encontraron diferencias estadísticas significativas en la eficacia del funcionamiento de los demás tiempos quirúrgicos. Las ventajas del ENdoAssist incluyo una respuesta y capacidad exacta para proveer al cirujano un completo control del area operativa sin la necesidad del asistente. Las desventajas incluyen el diseño y la necesidad de un pedal de activación.

\section{DISCUSIÓN}

Hasta el momento no existen un gran número de trabajos clínicos randomizados que comparen a la cirugía abierta con la cirugía laparoscopica y la cirugía laparoscopica robóticamente asistida. Si bien en algunos trabajos la cirugía robótica ha logrado equipararse a la cirugía abierta y laparoscópica, son necesarios aún sólidos resultados a largo plazo. Por otro lado, en las experiencias iníciales y desarrollo de la curva de aprendizaje de la cirugía robótica, aumenta el tiempo operatorio, lo que finalmente lleva a un mayor costo, más aún tomando en cuenta el costo original de la implementación robótica per se. 
Creemos que la cirugía robótica no representará un beneficio tangible para el paciente, hasta que en el tiempo se realicen estudios con mayor evidencia, que juzguen objetivamente a la cirugía robótica de la misma manera que sucedió con la cirugía laparoscópica tradicional.

A través de la revisión mencionada de la literatura, se pudo verificar de forma objetiva, la limitación de los reportes en cuanto a cirugía urológica robótica. En este sentido, sólo un pequeño número de estudios (4) cumplen con los criterios de un nivel de evidencia aceptable. La revisión realizada en Ingles se debe en parte a que la gran mayoría de trabajos relacionados con CUERA en nuestra búsqueda se encuentran en este idioma (366) comparados con los solos 20 artículos encontrados en idioma Español; ninguno de estos últimos con criterios de evidencia demostrable.

A partir de los resultados obtenidos en nuestra revisión podemos exhortar a realizar un esfuerzo aún mayor que se traduzca en una mayor cantidad de trabajos con alto nivel de evidencia con la finalidad de situar a la robótica como un estándar de oro en lo concerniente a procedimientos quirúrgicos en el campo de la urología. Los beneficios de la tecnología deben aprovecharse para mejorar los resultados de nuestros tratamientos, pero la evaluación objetiva de nuestras técnicas, permanece como elemento esencial de la evolución quirúrgica.

\section{CONCLUSIONES}

CURAE es una nueva opción quirúrgica urológica para cirugías como la prostatectomía radical, pieloplastia y cistectomía radical. Hay información con bajo nivel de evidencia que apoye la idea de la cirugía robótica como el próximo estándar de oro quirúrgico en urología. Existe una evolución quirúrgica en favor de los posibles beneficios de la tecnología robótica, pero hay necesidad de realizar estudios prospectivos controlados y randomizados en cirugía robótica urológica con el fin de consolidar la aplicación de esta novedosa tecnología.

\section{BIBLIOGRAFÍA y LECTURAS RECOMENDADAS (*lectura de interés $y^{* *}$ lectura fundamental)}

1. DAVIES, B.: "A review of robotics in surgery." Proc. Inst. Mech. Eng., 214: 129, 2000.

2. CHALLANCOMBE, B.J.; KHAN, M.S.; MURPHY, D. y cols.: "The history of robotics in urology". World J. Urol., 24: 120, 2006.
*3. MINH NGUYEN, M.; DAS, S.: "The evolution of robotic urologic surgery". Urol. Clin. N. Am., 31: 653, 2004.

4. CAMARILLO, D.B.; KRUMMEL, T.M.; SALISBURY, J.R.: "Robotic technology in surgery: past, present, and future". Am. J. Surg., 188: 2, 2004.

5. BINDER, J.; BRÄUTIGAM, R.; JONAS, D. y cols.: "Robotic surgery in urology: fact or fantasy?". BJU Int., 94: 1183, 2004.

6. HARRIS, S.J.; ARAMBULA-COSIO, F.; MEI, Q. y cols.: "The Probot-an active robot for prostate resection”. Proc. Inst. Mech. Eng., 211: 317, 1997.

*7. MARESCAUX, J.; LEROY, J.; RUBINO, F. y cols.: "Transcontinental robot-assisted remote telesurgery: Feasibility and potential applications". Ann. Surg., 235: 487, 2002.

8. ALBANI, J.M.: "The role of robotics in surgery". Mo. Med., 104: 166, 2007.

9. ELLISON, L.M.; PINTO, P.A.; KIM, F. y cols.: "Telerounding and patient satisfaction after surgery". J. Am. Coll. Surg., 199: 523, 2004.

10. HAILEY, D.; OHINMAA, A.; ROINE, R.: "Study quality and evidence of benefit in recent assessments of telemedicine". J. Telemed. Telecare., 10: 318, 2004.

11. DEMIRIS, G.; PATRICK, T.B.; MITCHELL, J.A. y cols.: "To telemedically err is human". Jt. Comm. J. Qual. Saf., 30: 521, 2004.

12. RIGBY, M.; FORSSTROM, J.; ROBERTS, R. y cols.: "Verifying quality and safety in health informatics services". BMJ., 323: 552, 2001.

*13. HERSH, W.R.; HELFAND, M.; WALLACE, J. y cols.: "Clinical outcomes resulting from telemedicine interventions: a systematic review". BMC Med. Inform. Decis. Mak., 1: 5, 2001.

*14. ROEHL, K.A.; HAN M.; RAMOS, C.G. y cols.: "Cancer progression and survival rates following anatomic radical retropubic prostatectomy in 3,478 consecutive patients: Long-term results”. J. Urol., 172: 910, 2004.

15. HAN, M.; PARTIN, A.W.; POUND, C.R. y cols.: "Longterm biochemical disease-free and cancerspecific survival following anatomic radical retropubic prostatectomy. The 15-year Johns Hopkins experience". Urol. Clin. North Am., 28: 555, 2001.

16. MIKHAIL, A.A.; ORVIETO, M.A.; BILLATOS, E.S. y cols.: "Robotic-assisted laparoscopic prostatectomy: First 100 patients with 1 year of follow up". Urology., 68: 1275, 2006.

*17. MENON, M.; SHRIVASTAVA, A.; KAUL, S. y cols.: "Vattikuti Institute prostatectomy: Contemporary technique and analysis of results". Eur. Urol., 51: 648, 2007.

*18. AHLERING, T.E.; SKARECKY, D.; LEE, D. y cols.: "Successful transfer of open surgical skills 
to a laparoscopic environment using a robotic interface: Initial experience with laparoscopic radical prostatectomy". J. Urol., 170: 1738, 2003.

19. PATEL, V.R.; TULLY, A.S.; HOLMES, R. y cols.: "Robotic radical prostatectomy in the community setting-the learning curve and beyond: Initial 200 cases". J. Urol., 174: 269, 2005.

20. TRABULSI, E.J.; GUILLONNEAU, B.: "Laparoscopic radical prostatectomy". J. Urol., 173: 1072, 2005.

21. MURPHY, D.; DASGUPTA, P.: "Robotic approaches to renal cancer". Curr. Opin. Urol., 17: 327, 2007.

22. ROGERS, C.G.; SINGH, A.; BLATT, A.M. y cols.: "Robotic partial nephrectomy for complex renal tumors: Surgical technique". Eur. Urol., 53: 514, 2008.

23. TÜRK, I.; DEGER, S.; WINKELMANN, B. y cols.: "Laparoscopic radical cystectomy with continent urinary diversion (rectal sigmoid pouch) performed completely intracorporeally: The initial 5 cases". J. Urol., 165: 1863, 2001.

24. GILL, I.S.; KAOUK, J.H.; MERANEY, A.M. y cols.: "Laparoscopic radical cystectomy and continent orthotopic neobladder performed completely intracorporeally: The initial experience". J. Urol., 168: 13, 2002.

25. CATHELINEAU, X.; ARROYO, C.; ROZET, F. y cols.: "Laparoscopic assisted radical cystectomy: The Mountsouris experience after 84 cases". Eur. Urol., 47: 780, 2005.

26. MENON, M.; HEMAL, A.K.; TEWARI, A. y cols.: "Nerve-sparing robot-assisted radical cystoprostatectomy and urinary diversion". BJU Int., 92: 232, 2003

27. HEMAL, A.K.; ABDOL-ENEIN, H.; TEWARI, A. y cols.: "Robotic radical cystectomy and urinary diversion in the management of bladder cancer”. Urol. Clin. North Am., 31: 719, 2004.

28. MILLER, N.L.; THEODORESCU, D.: "Status of robotic cystectomy in 2005". World J. Urol., 24: 180, 2006.

29. PESCHEL, R.; NEURURER, R.; BARTSCH, G. y cols.: "Robotic pyeloplasty: Technique and results". Urol. Clin. North Am., 31: 737, 2004.

30. JASPERS, J.E.; BREEDVELD, P.; HERDER, J.L. y cols.: "Camera and instrument holders and their clinical value in minimally invasive surgery”. Surg. Laparosc. Endosc. Percutan. Tech., 14: 145, 2004.

**31. ELLISON, L.M.; NGUYEN, M.: "Postoperative Robotic Telerounding: A Multicenter Randomized Assessment of Patient Outcomes and Satisfaction". Arch. Surg., 142: 1177, 2007.

**32. ELLISON, L.M.; PINTO, P.A.; KIM, F. y cols.: "Telerounding and Patient Satisfaction after Sur- gery”. J. Am. Coll. Surg., 199: 523, 2004.

33. GETTMAN, M.T.; BLUTE, M.L.: "Critical comparison of laparoscopic, robotic, and open radical prostatectomy: Techniques, outcomes, and cost". Curr. Urol. Rep., 7: 193, 2006.

34. ROZET, F.; HARMON, J.; CATHELINEAU, X. y cols.: "Robot - assisted versus pure laparoscopic radical prostatectomy". World J. Urol., 24: 171, 2006.

35. MENON, M.; SHRIVASTAVA, A.; TEWARI, A.: "Laparoscopic radical prostatectomy: Conventional and robotic". Urology., 66: 101, 2005.

36. STERRETT, S.; MAMMEN, T.; NAZEMI, T. y cols.: "Major urological oncological surgeries can be performed using minimally invasive robotic or laparoscopic methods with similar early perioperative outcomes compared to conventional open methods". World J. Urol., 25: 193, 2007.

37. LEPOR, H.: "Open versus robotic radical prostatectomy". Urol. Oncol., 24: 91, 2006.

38. BOCCON-GIBOD, L.: "Radical prostatectomy: open? Laparoscopic? Robotic?" Eur. Urol., 49: 598, 2006.

39. HERRELL, S.D.; SMITH, J.A. Jr.: "Laparoscopic and robotic radical prostatectomy: What are the real advantages?". BJU Int., 95: 3, 2005.

40. RASSWEILER, J.; HRUZA, M.; TEBER, D. y cols.: "Laparoscopic and robotic assisted radical prostatectomy-critical analysis of the results". Eur. Urol., 49: 612, 2006.

41. GUILLONNEAU, B.D.: "Laparoscopic versus robotic radical prostatectomy”. Nat. Clin. Pract. Urol., 2: 60, 2005.

42. JOSEPH, J.V.; VICENTE, I.; MADEB, R. y cols.: "Robot-assisted vs pure laparoscopic radical prostatectomy: Are there any differences?" BJU Int., 96: 39, 2005.

43. WANG, G.J.; BAROCAS, D.A.; RAMAN, J.D. y cols.: "Robotic vs open radical cystectomy: Prospective comparison of perioperative outcomes and pathological measures of early oncological efficacy”. BJU Int., 101: 89, 2008.

44. LINK, R.E.; BHAYANI, S.B.; KAVOUSSI, L.R.: "A prospective comparison of robotic and laparoscopic pyeloplasty”. Ann. Surg., 243: 486, 2006.

**45. CHALLACOMBE, B.; PATRICIU, A.; GLASS, J. y cols. "A randomized controlled trial of human versus robotic and telerobotic Access to the kidney as the first step in percutaneous nephrolithotomy". Comput Aided Surg., 10: 165, 2005.

**46. ANDREW, A.W.; IOANNIS, M.V.; RICHARD, E.: "Link Comparision of surgical performance during laparoscopic radical prostatectomy of two robotic camara holders, EndoAssist and AESOP: A Pilot Study”. Urology., 68: 70, 2006. 